\title{
Similarity Design Method of the Inflatable Buffer Landing System
}

\author{
Cheng He ${ }^{D},{ }^{1}$ Huan He $\mathbb{D}^{1},{ }^{1}$ and Tao Wang ${ }^{2}$ \\ ${ }^{1}$ State Key Laboratory of Mechanics and Control of Mechanical Structures, Nanjing University of Aeronautics and Astronautics, \\ Nanjing 210016, China \\ ${ }^{2}$ Key Laboratory of Advanced Technology of Small and Medium-Sized UAV, Ministry of Industry and Information Technology, \\ Nanjing University of Aeronautics and Astronautics, Nanjing 210016, China
}

Correspondence should be addressed to Huan He; hehuan@nuaa.edu.cn

Received 1 June 2019; Accepted 8 October 2019; Published 11 November 2019

Academic Editor: Paolo Gasbarri

Copyright @ 2019 Cheng He et al. This is an open access article distributed under the Creative Commons Attribution License, which permits unrestricted use, distribution, and reproduction in any medium, provided the original work is properly cited.

\begin{abstract}
The inflatable buffer landing system, such as the airbag, has been widely used for deep space exploration as a low-cost entry. The impact performance of an airbag landing attenuation system on Mars had to be proven through cushion testing on Earth. In this paper, a design method for the airbag landing attenuation system on Earth is proposed based on similarity relation. With this method, the impact response of the spherical airbag landing attenuation system is derived based on assumptions, and the principal factors have great influence on the impact response of the landing system have been proved. Then, the similarity relation between the full-scale airbag landing attenuation system in the Martian atmosphere and its Earth prototype is obtained through theoretical derivation. Finally, the proposed similarity relationship has been tested on several ground prototypes by FEA. The results of the study show that the Earth prototype by the developed design method is capable of predicting the impact response with good accuracy.
\end{abstract}

\section{Introduction}

The safety of the landing attenuation system of an interstellar probe is one of the most important technologies for deep space exploration. In order to ensure a successful landing and guarantee the reliability of the carried equipment, the design of a suitable landing attenuation system is necessary. Among all the existing landing attenuation systems, the airbag is the most widely used with wide adaptability. Hence, there have been several successful applications such as the "Mars Pathfinder," "Spirit," and "Opportunity" based on the omnidirection nonvented airbag landing attenuation system [1-3]. This kind of attenuation system is not sensitive to the orientation of the payload on landing, and it allows successful landings on a wider range of surfaces or under difficult conditions [4].

It is well known that the method to evaluate the performance of the airbag systems can be classified into two main groups: seeking the aid of finite element analysis programs designed for attenuation system or carrying out impact tests by prototype directly. For the former, there are plenty of dynamics simulations that are presented. He et al. [5] investigated an impact FE model for an airbag landing buffer system. Timmers et al. [6] carried out simulation and experimental studies surrounding a second generation of the airbag design developed by ILC Dover, building off of relevant first-generation design, analysis, and testing efforts. Wang et al. [7] analyzed the deployment and buffer process of the airbag system using FE based on a single-point Gaussian integral to improve the calculation efficiency. Compared to simulation methods, impact tests are more reliable to verify the effectiveness of the airbag system in actual work environment. Hence, numerous researchers have tried to characterize the performance of the inflatable landing attenuation system using impact tests [8-10]. Stein and Sandy [11] performed the impact testing at NASA's Plum Brook SPF and B2 Facilities. The Martian atmosphere (such as pressure, temperature, and gravity) was simulated, and an adjustable impact platform was used to allow different impact angles. Waye and Cole [12] conducted several freefall tests at several representative velocities in Sandia's High Altitude Chamber to demonstrate the structural integrity of the designed airbag 


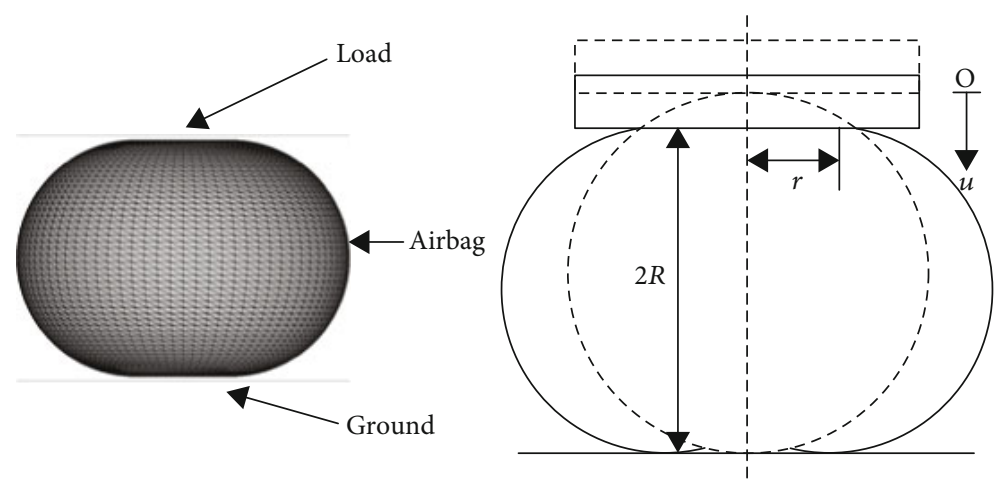

FIGURE 1: Schematic diagram of geometric changes in the spherical airbag cushioning process.

system. The results showed that the airbag impact attenuation design could protect the lander upon impact with the Martian surface.

However, the atmosphere of Mars is quite different from that of Earth; how to design the prototypes of the inflatable landing attenuation system and how to estimate impact response of the full-scale inflatable attenuation system in the Martian atmosphere using the impact response of the prototype in the Earth's atmosphere are vital for space exploration tasks. To overcome these two problems, it is necessary to research the similarity of the impact response for the deep space exploration inflatable landing attenuation system. Although considerable research has been devoted to dynamic similarity of structural aeroelastic characteristics [13-15], considerably less attention has been paid to similarity of the impact response of inflatable landing attenuation.

In this paper, the similarity between the impact responses of the deep space exploration inflatable cushion landing system and its prototype is discussed. In Section 2, the impact response of the inflatable landing cushion system with the parameters is derived; the similarities of the impact response are proposed in Section 3. In Section 4, the impact response of the full-scale inflatable landing attenuation system in the Martian atmosphere is estimated using the derived similarity relationships and the impact response of its Earth prototypes. Finally, the estimated results are validated by some numerical examples.

\section{Dynamic Equations of the Inflatable Landing System}

Consider that the nonvented spherical airbag contains $n$ moles of gas. The gas inside the airbag is regarded as the ideal gas and can be described by using the ideal gas equation:

$$
n e(\gamma-1)=P V
$$

where $e$ is the gas specific internal energy, $n$ is the mass of air in the airbag, $\gamma$ is the Gamma constant, $V$ is the gas volume, and $P$ is the internal pressure.

Given that the mass of the lander on the top of the nonvented airbag is $m$, the ambient pressure is $P_{\mathrm{e}}$ and the additional stiffness of the airbag is $k$. For simplicity, the positive direction of the lander's displacement is set as upward and normal to the landing surface. The mass of the gas contained in the airbag is neglected.

During the landing process, the contact between the bottom of the airbag and the landing surface is continuous, and the lateral expansion of the airbag and the effect of the variation of the contact area are also neglected; the deformation of the airbag cross-section is shown in Figure 1.

Setting the equilibrium position as the initial position, at time $t$, the displacement of the lander is $u$. Based on the above assumptions, using the second law of Newton, the equation of movement describing the lander can be written as

$$
m \ddot{u}+\left(P_{\mathrm{e}}-P\right) S+k u-f_{0}=m g \text {, }
$$

where $S$ is the contact area between the lander and the airbag and $f_{0}$ is the support force exerted on the lander by the fabric at equilibrium position.

Assume the initial parameters of the airbag before contact with landing surface as follows: the initial gas specific internal energy inside the airbag is $e_{0}$, the initial volume of the airbag is $V_{0}$, the initial internal pressure is $P_{0}$, and the initial radius of the spherical airbag is $R_{0}$. During that period, the landing system is in a state of freefall so the acceleration of the lander is equal to the gravitational acceleration.

Regarding the initial deformation of the spring as the origin point, then

$$
\left(P_{\mathrm{e}}-P_{0}\right) S=f_{0}
$$

As shown in Figure 1, the cross-section length of the airbag during the buffering process is assumed to be constant. Hence,

$$
l=2 \pi R_{0}
$$

Then, we can conclude as follows at any time:

$$
l=\pi\left(2 R_{0}-u\right)+4 r
$$

Combining Equations (4) and (5), we can draw that $r=\pi u / 4$. 
After the airbag makes contact with the landing surface, the volume of the gas inside the airbag at any time can be expressed as

$$
V=\pi\left(2 R_{0}+u\right)\left(\frac{\pi u}{4}\right)^{2}+\pi^{2}\left(\frac{\pi u}{4}\right)\left(2 R_{0}+\frac{u}{2}\right)^{2}+\frac{4}{3} \pi\left(R_{0}+\frac{u}{2}\right)^{3} .
$$

Substituting Equation (6) into Equation (1), the internal pressure of the airbag is obtained:

$$
P=\frac{n e_{0}(\gamma-1)}{V}
$$

Thus, the internal pressure inside the airbag is a function which has a single variable, the displacement $u$.

Using the Taylor expansion at the initial position, where $u=0$, the internal pressure can be expressed as

$$
P=\frac{3 n e_{0}(\gamma-1)}{4 \pi R_{0}^{3}}+\frac{9 n e_{0}(\gamma-1)\left(8-\pi^{2}\right)}{64 \pi R_{0}^{4}} u+\mathrm{O}\left(u^{2}\right) .
$$

Moreover,

$$
V_{0}=\frac{4 \pi R_{0}^{3}}{3}
$$

Substituting Equation (9) into Equation (8) and omitting the items higher than the second order

$$
P=\frac{n e_{0}(\gamma-1)}{V_{0}}\left(1+\frac{3\left(8-\pi^{2}\right)}{16 R_{0}} u\right) .
$$

Substituting Equation (10) into Equation (2), the equation of movement can be written as

$$
m \ddot{u}+P_{\mathrm{e}} S-\frac{n e_{0}(\gamma-1)}{V_{0}}\left(1+\frac{3\left(8-\pi^{2}\right)}{16 R_{0}} u\right) S+k u-f_{0}=m g .
$$

At the initial moment, the internal pressure is

$$
P_{0}=P(0)=\frac{n e_{0}(\gamma-1)}{V_{0}}
$$

Substituting Equation (12) into Equation (11), and then combining Equation (3), the simplified equation of movement can be expressed as

$$
m \ddot{u}+\left(\frac{3\left(\pi^{2}-8\right)}{16 R_{0}} P_{0} S+k\right) u=m g \text {. }
$$

For the inflatable landing system, the support force is almost entered by the gas contained in the airbag. The additional stiffness of the airbag is related to the lateral expansion during the compression and usually satisfies

$$
k \ll \frac{3\left(\pi^{2}-8\right)}{16 R_{0}} P_{0} S .
$$

So Equation (13) can be simplified as

$$
m \ddot{u}+\frac{3\left(\pi^{2}-8\right)}{16 R_{0}} P_{0} S u=m g .
$$

From Equation (15), we can get the natural frequency of the system:

$$
\omega=\sqrt{\frac{3\left(\pi^{2}-8\right)}{16 R_{0} m} P_{0} S} .
$$

Given that at the moment when the contact begins, the lander has the following initial condition:

$$
\begin{aligned}
& u(0)=0, \\
& \dot{u}(0)=v_{0} .
\end{aligned}
$$

Then, the displacement response of the lander can be calculated as

$$
u=\frac{v_{0}}{\omega} \sin \omega t+\frac{g}{\omega^{2}}(1-\cos \omega t) .
$$

Differentiating $u$ yields

$$
\begin{aligned}
& \dot{u}=v_{0} \cos \omega t+\frac{g}{\omega} \sin \omega t, \\
& \ddot{u}=-\omega v_{0} \sin \omega t+g \cos \omega t .
\end{aligned}
$$

Alternatively, Equation (20) can be written as

$$
\ddot{u}=\sqrt{\left(\omega v_{0}\right)^{2}+g^{2}} \sin (\omega t+\varphi),
$$

where $\tan \varphi=g / \omega v_{0}$

For most airbag landing attenuation systems, for the relationship where $\omega v_{0} \gg g$ is assured, $\ddot{u}$ can be approximately expressed as

$$
\ddot{u} \approx v_{0} \omega \sin \omega t \text {. }
$$

Thus, we can get the maximum landing impact acceleration:

$$
\ddot{u}_{\max }=v_{0} \omega=v_{0} \sqrt{\frac{3\left(\pi^{2}-8\right)}{16 R_{0} m} P_{0} S}=c_{0} v_{0} \sqrt{\frac{P_{0} S}{R_{0} m}},
$$

where $c_{0}=\sqrt{3\left(\pi^{2}-8\right) / 16}$. From Equation (23), it is obvious that the peak overloading of the landing system is related to several factors, such as the landing velocity, the mass of the lander, the internal pressure of the airbag, the gas volume inside the airbag, and the contact area. Besides, Equation (23) can also be used to offer some reference to the design of the inflatable landing attenuation system. 
Omitting the subscripts in Equation (23) for simplicity, it can be expressed as

$$
\ddot{u}_{\max }=v \omega=c_{0} v \sqrt{\frac{P S}{R m}} .
$$

\section{Dynamic Similarities}

From Equation (24), the maximum accelerations of the prototypes and the full-scale landing system can be expressed as

$$
\begin{aligned}
& a_{\mathrm{P}}=c_{0} v_{\mathrm{P}} \sqrt{\frac{P_{\mathrm{P}} S_{\mathrm{P}}}{R_{\mathrm{P}} m_{\mathrm{P}}}}, \\
& a_{\mathrm{F}}=c_{0} v_{\mathrm{F}} \sqrt{\frac{P_{\mathrm{F}} S_{\mathrm{F}}}{R_{\mathrm{F}} m_{\mathrm{F}}}},
\end{aligned}
$$

where $a_{\mathrm{P}}$ is the maximum acceleration of the prototype under a certain environment and $a_{\mathrm{F}}$ is the maximum acceleration of the full-scale landing system under real Martian environment; the subscripts "P" and " $F$ " represent the prototype and the full-scale model, respectively.

The ratio of $a_{\mathrm{P}}$ and $a_{\mathrm{F}}$ is

$$
\frac{a_{\mathrm{P}}}{a_{\mathrm{F}}}=\frac{v_{\mathrm{P}} \omega_{\mathrm{P}}}{v_{\mathrm{F}} \omega_{\mathrm{F}}}=\frac{\left(v_{\mathrm{P}} / v_{\mathrm{F}}\right) \sqrt{S_{\mathrm{P}} / S_{\mathrm{F}}} \sqrt{P_{\mathrm{P}} / P_{\mathrm{F}}}}{\sqrt{m_{\mathrm{P}} / m_{\mathrm{F}}} \sqrt{R_{\mathrm{P}} / R_{\mathrm{F}}}} .
$$

Considering that the ground prototype and the full-scale inflatable system have the same landing velocity, $\lambda, h$, and $\eta$ are the geometric ratio, initial internal pressure ratio, and mass ratio of the two models, respectively. All the parameters have the following relationships:

$$
\begin{aligned}
& R_{\mathrm{P}}=\lambda R_{\mathrm{F}}, \\
& S_{\mathrm{P}}=\lambda^{2} S_{\mathrm{F}}, \\
& P_{\mathrm{P}}=h P_{\mathrm{F}}, \\
& m_{\mathrm{P}}=\eta m_{\mathrm{F}} .
\end{aligned}
$$

Substituting Equation (27) into Equation (26), the ratio of $a_{\mathrm{P}}$ and $a_{\mathrm{F}}$ can be expressed as

$$
\frac{a_{\mathrm{P}}}{a_{\mathrm{F}}}=\sqrt{\frac{h \lambda}{\eta}}
$$

The above equation gives the relationship between the maximum accelerations of the ground prototype and the full-scale inflatable landing system during the landing.

With Equation (22), the moment when the acceleration becomes maximum can be obtained as

$$
t=\frac{\pi}{2 \omega}
$$

Similarly, the relationship between the moments when accelerations of the ground prototype and the full-scale inflatable landing system become maximal during the landing process can be expressed as

$$
\frac{t_{\mathrm{P}}}{t_{\mathrm{F}}}=\frac{\omega_{\mathrm{F}}}{\omega_{\mathrm{P}}}=\sqrt{\frac{\eta}{h \lambda}} .
$$

\section{Discussions on the Similarities of Inflatable Landing System in the Martian Atmosphere}

Based on the relationships discussed above, one can try to design the ground prototype of the inflatable system using the full-scale inflatable landing system in the Martian atmosphere. In this paper, two approaches as follows are considered.

(a) Setting the geometric ratio of the prototype and the full-scale system to be the ratio of the accelerations of gravity on Mars and Earth

$$
\lambda=\frac{g_{\mathrm{F}}}{g_{\mathrm{P}}}=0.38 .
$$

Assuming the ratio of the initial internal pressure of the two models to be $h$, the mass ratio of the two models $\eta$ is designed to be

$$
\eta=h \lambda^{3}
$$

For the prototype designed as above, using Equation (28), once the maximum acceleration $a_{\mathrm{P}}$ and the corresponding moment $t_{\mathrm{P}}$ during the landing procedure are obtained, the maximum acceleration $a_{\mathrm{F}}$ and the corresponding moment $t_{\mathrm{F}}$ of the full-scale inflatable landing system in the Martian atmosphere can be calculated as

$$
\begin{gathered}
a_{\mathrm{F}}=\lambda a_{\mathrm{P}}, \\
t_{\mathrm{F}}=\frac{t_{\mathrm{P}}}{\lambda} .
\end{gathered}
$$

(b) First, determine the mass of the prototype based on the practical experiment condition, then set the initial internal pressure ratio to be $h$; the geometric ratio of the two models is then designed to be

$$
\lambda=\frac{\eta}{h}
$$

Combining Equations (34) and (28), when the maximum acceleration $a_{\mathrm{P}}$ and the corresponding moment $t_{\mathrm{P}}$ during the landing process are obtained from drop tests, the maximum acceleration $a_{\mathrm{F}}$ and 


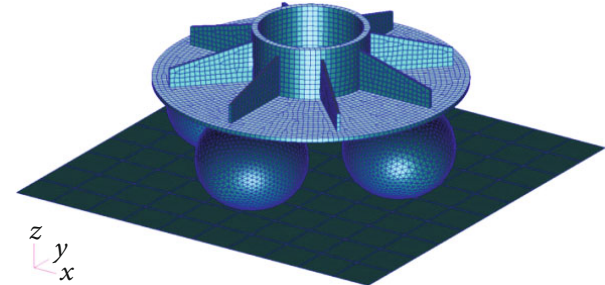

FIGURE 2: FE model of inflatable cushion system.

the corresponding moment $t_{\mathrm{F}}$ of the full-scale inflatable landing system in the Martian atmosphere can be calculated as

$$
\begin{gathered}
a_{\mathrm{F}}=a_{\mathrm{P}}, \\
t_{\mathrm{F}}=t_{\mathrm{P}} .
\end{gathered}
$$

\section{Numerical Examples}

For the two impact dynamic models of the landing system, the response calculated by a nonlinear transient finite element analysis program MSC.Dytran is regarded as the accurate results to verify the results obtained using dynamic similarity. The difference is that the former is in line with the theoretically derived spherical airbag model, while the latter buffer system, as the well-known Mars Pathfinder System, uses a spherical-like configuration, and its geometry is more complex.

5.1. Example I. The full-scale inflatable landing attenuation system in the Martian atmosphere is regarded as the reference system. The inflatable system consists of four spherical airbags with a radius of 480 millimeters, the mass of the landing attenuation systems is $50 \mathrm{~kg}$, the initial internal pressure of the airbag is $3200 \mathrm{~Pa}$, the Martian gravity is $3.72 \mathrm{~m} / \mathrm{s}^{2}$, and the landing velocity of the system is $5 \mathrm{~m} / \mathrm{s}$.

MSC.Dytran was used to compute the impact overloading for the inflatable landing buffer system for the FE model, where rigid elements were used to model the landing attenuation systems and the membrane elements were used to model the airbag fabric. The connection between the airbag and the lander is simulated by sharing nodes of local elements. The air contained within the bags was assumed to behave as an adiabatic, isentropic ideal gas, with uniform pressure and temperature throughout the airbag before impact. The master-slave contact algorithm was used to simulate the interaction between the airbag model and the rigid landing surface. The developed FE model for the inflatable landing buffer system is shown in Figure 2. In this section, three different prototypes were considered; the specific scale factors are shown in Table 1.

According to the parameters listed in Table 1, the model parameters of the ground prototypes were calculated from Equation (27). For prototype I, the gravity equals to $3.72 \mathrm{~m} / \mathrm{s}^{2}$, the same as the Martian gravity. According to the scale factors, the gravity for model II and model III was $9.80 \mathrm{~m} / \mathrm{s}^{2}$, equal to Earth's gravity. Based on the relationships
TABLE 1: Scale factors of the prototypes.

\begin{tabular}{lcc}
\hline Case & $n$ & $\lambda$ \\
\hline Prototype I & 2.0 & 1.0 \\
Prototype II & 1.0 & 0.38 \\
Prototype III & 2.0 & 0.38 \\
\hline
\end{tabular}

TABLe 2: Mechanical characteristics of the prototypes.

\begin{tabular}{lccc}
\hline Case & $R(\mathrm{~mm})$ & $m(\mathrm{~kg})$ & $p_{0}(\mathrm{~Pa})$ \\
\hline Prototype I & 480 & 100.0 & 6400.0 \\
Prototype II & 182.4 & 2.744 & 3200.0 \\
Prototype III & 182.4 & 5.487 & 6400.0 \\
\hline
\end{tabular}

given in Table 1, the mechanical characteristics of the prototype can be obtained as shown in Table 2.

Except the parameters shown in Table 1, the three FE models of the prototypes were the same as the full-scale model and so was the landing velocity. Assuming that the simulation results obtained from the full-scale model can reflect the real landing process in the Martian atmosphere, the maximum impact overloading and its corresponding moment could be regarded as the correct value.

According to Equation (34), the maximum impact overloading, the peak times, and the pulse width of the fullscale model in the Martian atmosphere can be calculated using the data obtained from the scaled models, and the results were called estimation results of the scaled models. The errors between the estimation results using the scaled models and the full-scale model were defined as $\varepsilon=|f-\bar{f}| /$ $\bar{f} \times 100 \%$. Figure 3 compares the impact overloading curves of the full-scale model and the three prototype models before and after transformation by Equation (33). Meanwhile, we compare the prototype prediction results at peak overloading value, peak times, and pulse width in Table 3 . It is seen from Figure 3 and Table 3 as follows:

(1) We compare the prototype prediction results at peak overloading value and peak times in Table 3, where "g" was set as $9.80 \mathrm{~m} / \mathrm{s}^{2}$. Since the landing impact overload of the model in Table 3 is greater than environmental gravity, it can be concluded that neglecting the influence of the ambient gravity is acceptable

(2) The difference of the prototype predictive ability is closely related to the full-scale system. For example, the error in the peak overloading for prototypes I and III does not exceed 2.5\%; however, it sharply increases to around $7.3 \%$ for prototype II. A similar trend is seen for the error of peak times and pulse width for prototypes I, II, and III. So we conclude that the prototype based on models I and III can give a better estimation of the impact overloading for the full-scale inflatable landing system in the Martian atmosphere

(3) The influence of the additional stiffness of the airbag is the first reason for the errors; neglecting the 


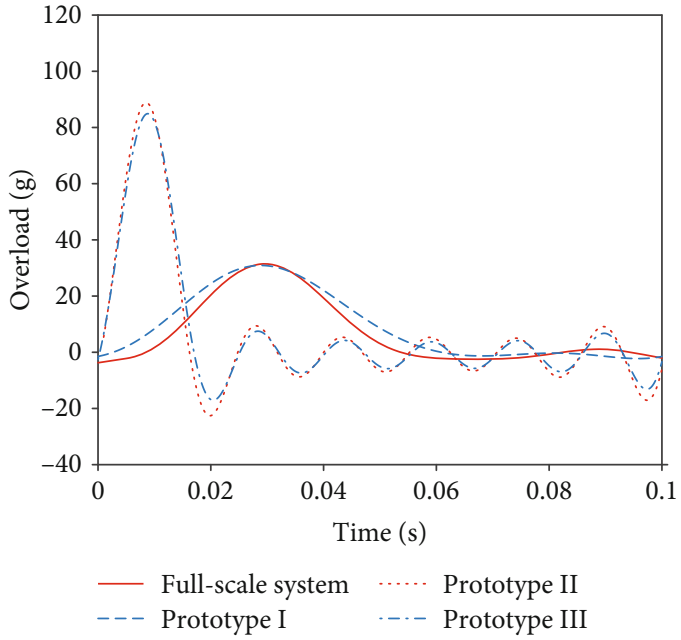

(a) Before transfer

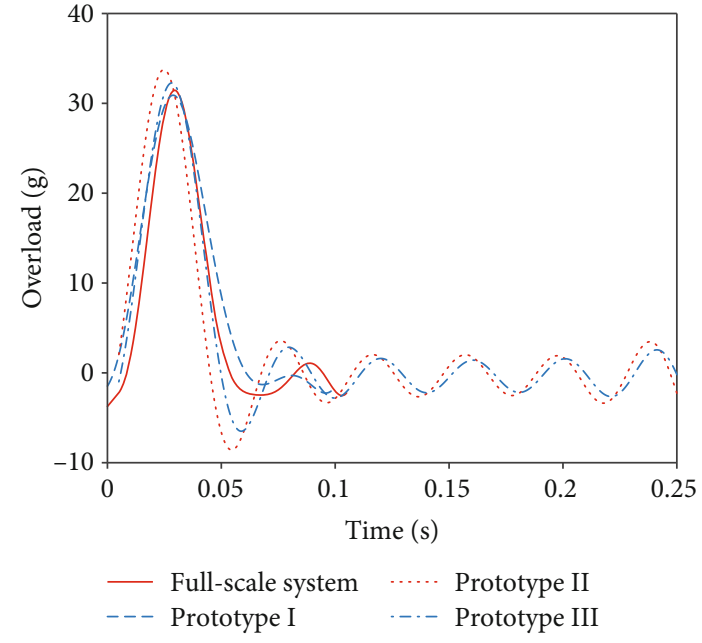

(b) After transfer

FIGURE 3: Comparison of the overloading.

TABLE 3: Comparison of the overloading for the full-scale system and the prototype.

\begin{tabular}{|c|c|c|c|c|c|c|}
\hline Case & Peak overloading (g) & Error (\%) & Peak times (s) & Error (\%) & Pulse width (s) & Error (\%) \\
\hline Full scale & 31.44 & - & 0.0298 & - & 0.058 & - \\
\hline Prototype I & 30.89 & 1.7 & 0.0292 & 2.0 & 0.063 & 8.6 \\
\hline Prototype II & 88.83 & 7.3 & 0.026 & 12.7 & 0.061 & 5.2 \\
\hline Prototype III & 84.84 & 2.5 & 0.0284 & 6.4 & 0.0596 & 2.8 \\
\hline
\end{tabular}
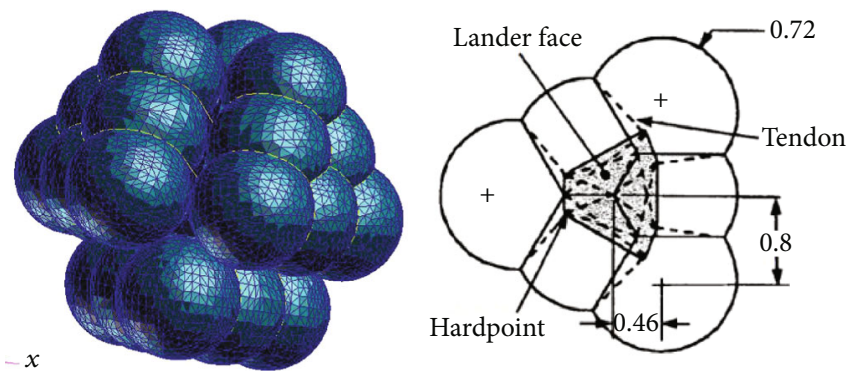

Figure 4: Model of the Mars Pathfinder airbags.

nonlinear influence during the compression of the airbag is the second one. Both the Taylor expansion of the internal pressure around the equilibrium position and the lateral expansion coefficients could have influence on the impact responses of the landing system. As shown in Table 3, the pressure in prototypes I and III is higher than in prototype II. Obviously, the higher initial pressure, the more accurate the approximation for Equation (14), which leads to solutions that are closer to reality

5.2. Example II. Similar to example I, a full-scale impact FE model of the inflatable landing attenuation system ("Mars Pathfinder" shown in Figure 4) in the Martian atmosphere has been formulated. The mass of the landing attenuation systems is $150 \mathrm{~kg}$, the initial internal pressure of the airbag is $3200 \mathrm{~Pa}$, the landing velocity of the system is $17.3 \mathrm{~m} / \mathrm{s}$,

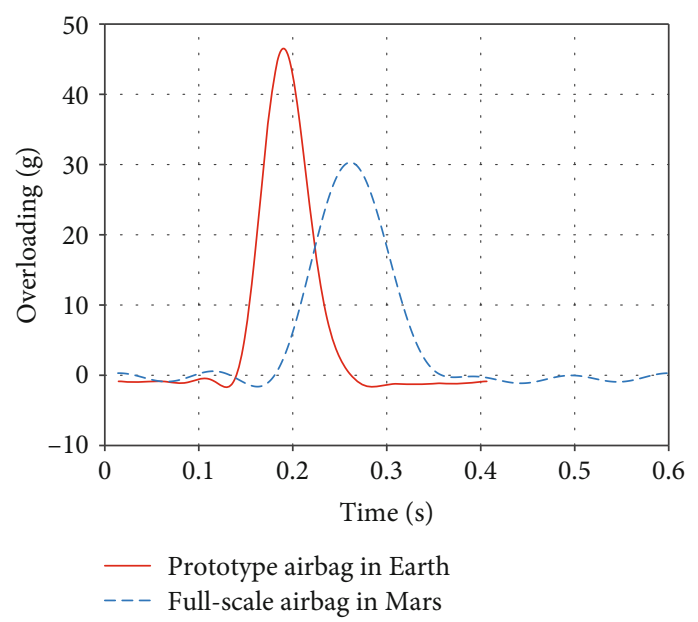

FIgURE 5: Comparison of the overloading between the full-scale system and the prototype.

and the atmosphere parameters are the same as the model used in example I.

The mass of the Earth prototype is $60.0 \mathrm{~kg}$, the radius of the spherical airbag is $0.529 \mathrm{~m}$, and the initial internal pressure is $3200 \mathrm{~Pa}$. Figure 5 gives the comparison of the overloading between the prototype and the full-scale model. We found that the calculated peak overloading of the prototype is $46.03 \mathrm{~g}$, and the peak time is $0.1930 \mathrm{~s}$.

Based on Equation (29), the ratios between the two models are as follows: $h=1.0, \lambda=0.735$, and $\eta=0.4$. According to the similarity relationships showed in Equations (28) 
TABLE 4: Comparison of the overloading of the prototype and the full-scale system for the Mars Pathfinder.

\begin{tabular}{lcccccc}
\hline Case & Peak overloading (g) & Error (\%) & Peak times (s) & Error (\%) & Pulse width (s) & Error (\%) \\
\hline Full scale & 30.92 & - & 0.2653 & - & 0.205 & - \\
Prototype & 33.85 & 9.48 & 0.2615 & 1.43 & 0.2031 & 0.92 \\
\hline
\end{tabular}

and (30), the ratio coefficients of the peak overloading and its peak times can be calculated as

$$
\begin{aligned}
& \frac{a_{\mathrm{P}}}{a_{\mathrm{F}}}=\sqrt{\frac{h \lambda}{\eta}}=1.36, \\
& \frac{t_{\mathrm{P}}}{t_{\mathrm{F}}}=\sqrt{\frac{\eta}{h \lambda}}=0.738 .
\end{aligned}
$$

Using the above ratio coefficients and the impact results of the prototypes, the impact result of the full-scale model can be estimated, and the impact overloading is compared in Table 4 . We found that the errors of peak value, peak times, and pulse width are just $9.48 \%, 1.43 \%$, and $0.92 \%$, respectively, which means the similarity relationships proposed in the paper are acceptable.

\section{Conclusion}

In this paper, the peak overloading of the inflatable landing system was derived from the equilibrium equation of the lander based on some assumptions. It was found that the landing velocity, the mass of the lander, the initial internal pressure, the gas volume, and the contact area are all essential to the peak overloading. The relationship derived here can be a reference for the design of the inflatable landing attenuation system.

Based on the similarity relationship, a method to estimate the impact response of the full-scale inflatable landing system in the Martian atmosphere using the simulated data of the ground prototype was proposed.

Several prototypes were designed based on different design ideas. The results of the numerical examples illustrated the validity of the proposed similarity relationship. The model which can represent the impact response of the full-scale inflatable landing attenuation system in the Martian atmosphere was also given.

\section{Data Availability}

The [model for Example.zip] data used to support the findings of the manuscript (titled "Similarity Design Method of the Inflatable Airbag Impact Attenuation System for Deep Space Exploration") have been deposited in the "figshare" repository (10.6084/m9.figshare.8214794).

\section{Conflicts of Interest}

The authors declare that they have no conflicts of interest.

\section{Authors' Contributions}

Cheng He coordinated the project and edited the manuscript, Huan $\mathrm{He}$ conduct the relationship between the impact response of the inflatable landing cushion system and the parameters of the airbag, and Tao Wang analyzed the data and contributed valuable discussion. All authors read and approved the final manuscript.

\section{Acknowledgments}

This paper is funded by the National Natural Science Foundation of China (Grant No. 11602105) and the Natural Science Foundation of Jiangsu Province of China (Grant No. BK20160782).

\section{References}

[1] D. Cadogan, C. Sandy, and M. Grahne, "Development and evaluation of the Mars Pathfinder inflatable airbag landing system," Acta Astronautica, vol. 50, no. 10, pp. 633-640, 2002.

[2] T. R. Smith, J. Ware, C. E. Willey, and C. Sandy, "Orion CEV earth landing impact attenuating airbags - design challenges and application," in 2007 IEEE Aerospace Conference, New York, 2007.

[3] R. Sakagami, R. Takahashi, A. Wachi et al., "Integral design method for simple and small Mars lander system using membrane aeroshell," Acta Astronautica, vol. 144, pp. 103-118, 2018.

[4] D. Northey and C. Morgan, "Improved inflatable landing systems for low cost planetary landers," Acta Astronautica, vol. 59, no. 8-11, pp. 726-733, 2006.

[5] H. He, Z. Chen, and C. He, "A hierarchical updating method for finite element model of airbag buffer system under landing impact," Chinese Journal of Aeronautics, vol. 28, no. 6, pp. 1629-1639, 2015.

[6] R. Timmers, R. Hardy, and J. Welch, "Modeling and simulation of the second-generation Orion Crew Module airbag landing system," in 20th AIAA Aerodynamic Decelerator Systems Technology, Seattle, Washington, 2009.

[7] H. Wang, Q. Rui, H. Hong, and J. Li, Airdrop Recovery Systems with Self-Inflating Airbag: Modeling and Analysis, John Wiley \& Sons, Singapore, 2017.

[8] P. Dreher, R. Canfield, and R. Maple, "Experimental dynamic response of a solid object to various diameter low pressure airbags," in 47th AIAA/ASME/ASCE/AHS/ASC Structures, Structural Dynamics, and Materials Conference, vol. 1, Newport, Rhode Island, 2006no. 7.

[9] E. L. Fasanella, "Multiterrain earth landing systems applicable for manned space capsules," Journal of Aerospace Engineering, vol. 22, no. 3, pp. 201-213, 2009.

[10] B. Tutt, C. Sandy, and J. Corliss, "Status of the development of an airbag landing system for the Orion Crew Module," in 20th 
AIAA Aerodynamic decelerator systems technology conference and seminar, Seattle, Washington, 2009.

[11] J. Stein and C. Sandy, "Recent developments in inflatable airbag impact attenuation systems for Mars exploration," in 44th AIAA/ASME/ASCE/AHS/ASC Structures, Structural Dynamics, and Materials Conference, Norfolk, Virginia, 2003.

[12] D. E. Waye and J. Kenneth Cole, "Mars Pathfinder airbag impact attenuation system," in 13th Aerodynamic Decelerator Systems Technology Conference, Clearwater beach, FL, 1995.

[13] L. G. Horta and R. G. Kvaternik, A historical perspective on dynamics testing at the Langley Research Center, 2000.

[14] P. Pereira, L. Almeida, A. Suleman, V. Bond, R. Canfield, and M. Blair, "Aeroelastic scaling and optimization of a joinedwing aircraft concept," in 48th AIAA/ASME/ASCE/AHS/ASC Structures, Structural Dynamics, and Materials Conference, 2007.

[15] Q. Wu, Z. Wan, and C. Yang, "Design optimization of flutter scaled model considering structural dynamic and flutter constraints," Acta Aeronautica et Astronautica Sinica, vol. 32, no. 7, pp. 1210-1216, 2011. 


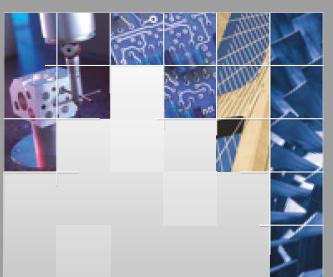

\section{Enfincering}
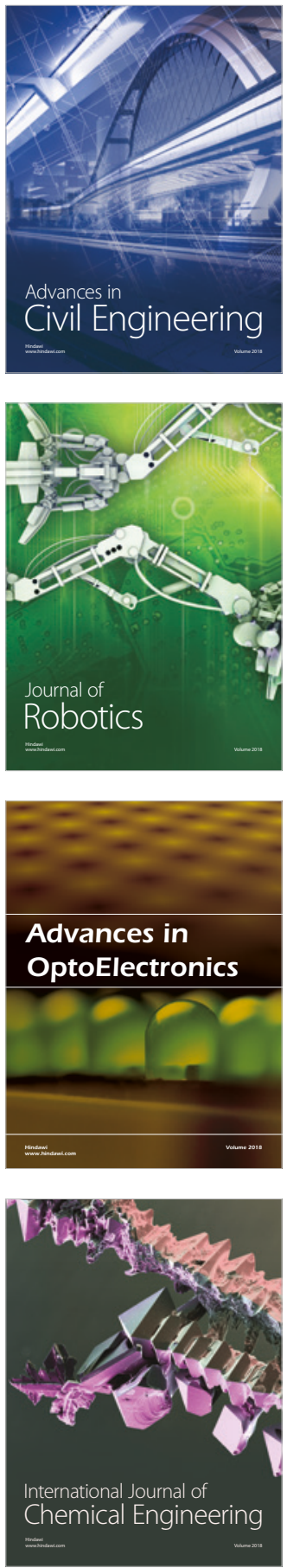

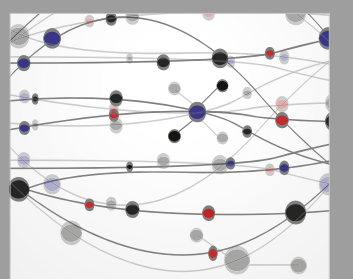

\section{Rotating \\ Machinery}

The Scientific World Journal

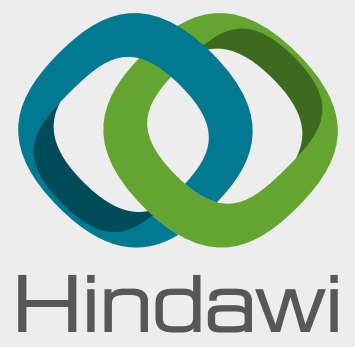

Submit your manuscripts at

www.hindawi.com
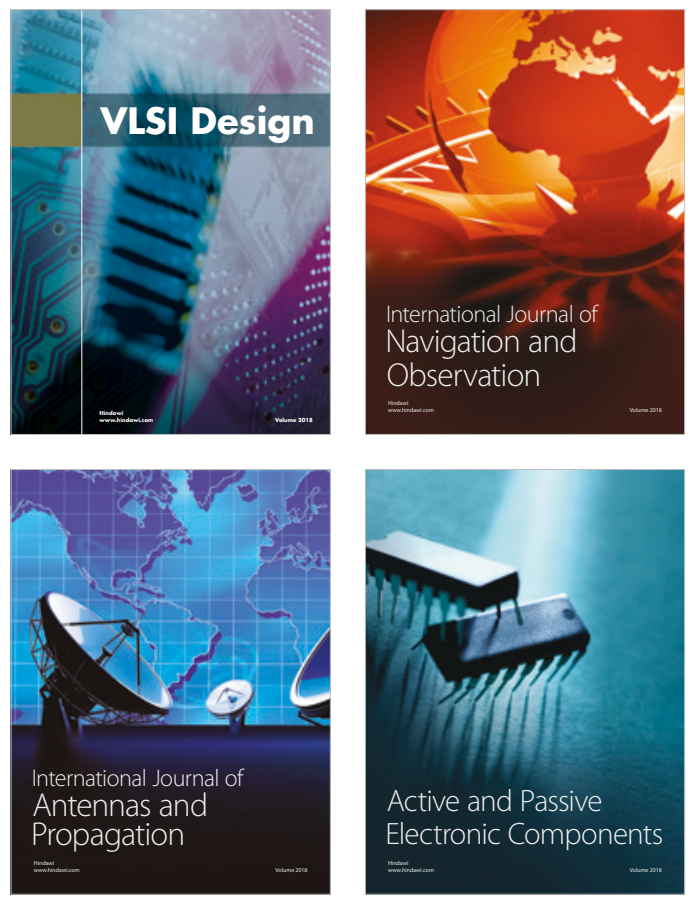
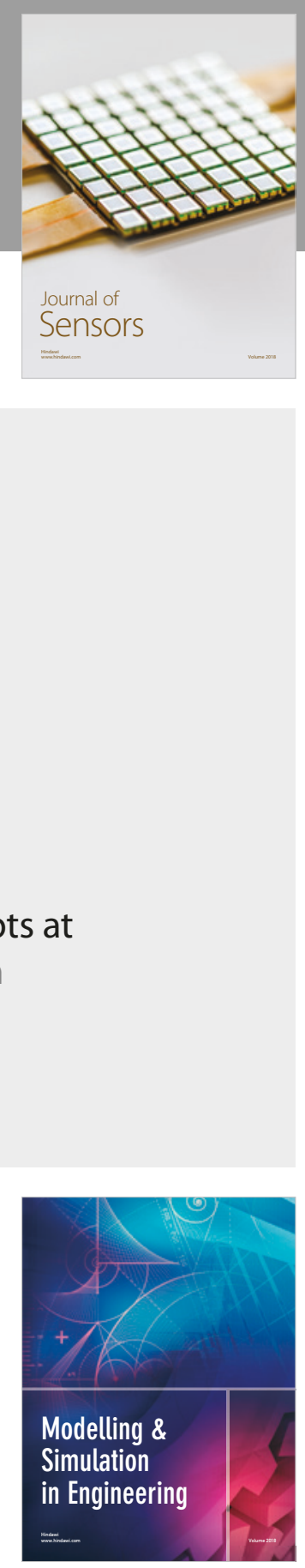

\section{Advances \\ Multimedia}
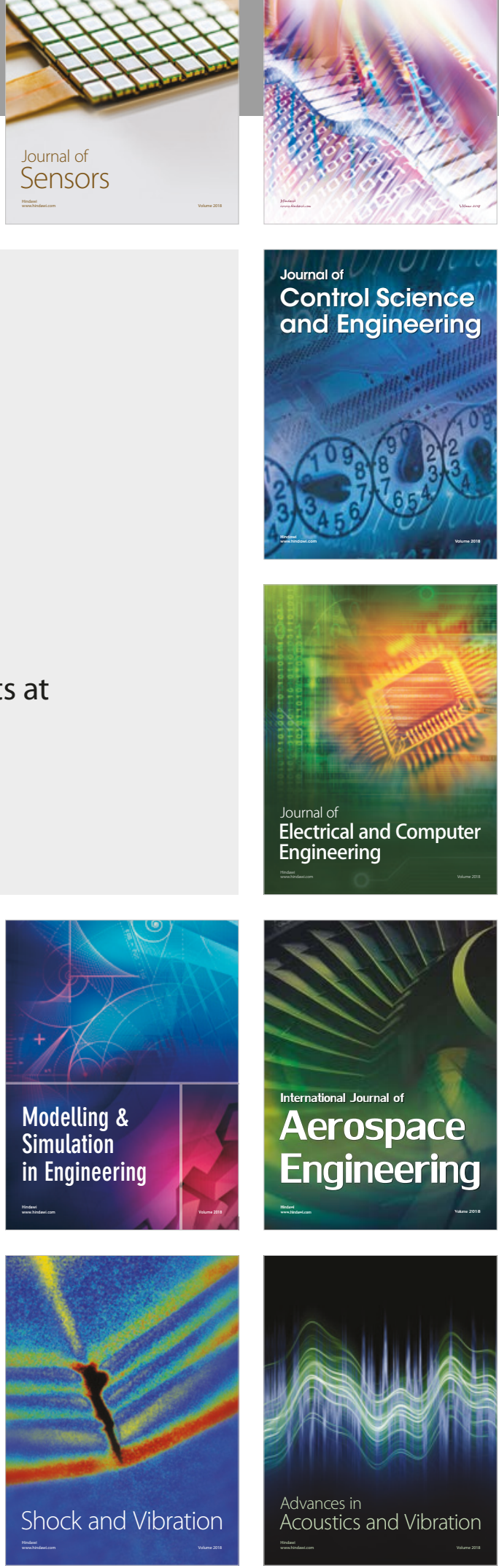